\title{
Dynamical Power Optimization by Decentralized Routing Control in Multi-Hop Wireless Control Networks *
}

\author{
M.D. Di Benedetto, A. D'Innocenzo, and E. Serra
}

\author{
Department of Electrical and Information Engineering, \\ Center of Excellence DEWS. University of L'Aquila - L'Aquila, Italy. \\ email:\{mariadomenica.dibenedetto,alessandro.dinnocenzo,emmanuele.serra\}@univaq.it
}

\begin{abstract}
We propose a mathematical and simulative framework for dynamic routing control in order to minimize power consumption objectives, which is compliant with the WirelessHART specification for multi-hop wireless control networks. To achieve this goal we propose the use of a decentralized optimization algorithm, which will be proved to be robust with respect to time-varying transmission channels and to node failures.
\end{abstract}

Keywords: Control of networks; Sensor networks; Complex system management.

\section{INTRODUCTION}

Wireless communication is emerging in control applications with main advantages being reduced installation costs and increased flexibility, as well as ease of maintenance, debugging and diagnostics. However, wireless devices are usually battery powered: for this reason, energy efficiency and power minimization is a fundamental issue to be addressed. Control with wireless technologies typically involves multiple communication hops for conveying information from wireless sensor devices to a controller, and from the controller to wireless actuator devices. The challenges in designing and analyzing multi-hop wireless control networks (WCN) are best explained by considering the recently developed WirelessHART (www.hartcomm2.org) Song et al. (2007) and ISA-100 (www.isa.org) specifications. These standards allow designers of wireless control networks to distribute a synchronous communication schedule to all nodes of the network, in order to orchestrate conveyance of sensing information to the controller and of control information to the actuators. More specifically, time is divided into slots of fixed length (10ms), and a periodic schedule is defined as an assignment of nodes to send data in each slot. The standard specifies a syntax for defining schedules and a mechanism to apply them. However, once defined a scheduling, the issue of designing routing remains undefined by the specification. More precisely, each node locally decides to which node transmitting data, among a predefined set of admissible destination nodes defined by the scheduling policy. To allow systematic methods for computing and validating local routing policies for each node and quantify their effect on the power consumption of the whole network, a rigorous mathematical model is needed. The routing problem is in fact crucial when dealing with WCN, because of the intrinsic unreliability of the nodes and of battery operation. In this context, multipath routing has been introduced in Mueller et al. (2003), Zaumen, W.T. and Garcia-Luna-Aceves, J.J. (1998) and Hazarika et al. (2004), allowing multiple paths between a single source and a single destination node to increase

* This research has been partially supported by the European Commission through the NoE HYCON2. the reliability of data, or to provide load balancing and energy conservation. In Mueller et al. (2003), the authors specifically examine the issues of multi-path routing on MANETs (Mobile Ad hoc NETworks). In Hazarika et al. (2004) the key idea is to route flows so that links with less interference and hence lower power requirements are better utilized. In Zaumen, W.T. and Garcia-Luna-Aceves, J.J. (1998) a new distributed algorithm for the dynamic computation of multiple loop-free paths from source to destination in a computer network is presented. In wired networks, routing protocols are usually based on link state or distance vector routing algorithms. The link state (e.g. Dijkstra algorithm Hedrick (1988)) is a centralized routing algorithm in which each node has the same knowledge of the other nodes. The distance vector algorithm (e.g. one based on the classical Bellman-Ford shortest-path algorithm Moy (1991)) is a decentralized algorithm in which each node communicates only with its neighbors. They both search for the shortest path from the source node to the destination node. Naturally, these conventional algorithms are not efficient in wireless networks, since the shortest path solution is neither the most robust nor the one that optimally preserves the battery of each node. Single path routing solutions have been proposed in Perkins, C.E. and Royer, E.M. (1999) and Johnson, D.B. and Maltz, D.A. (1996). In the context of wired networks the fundamental issues of congestion and flow control are discussed in Bertsekas and Gallager (1987). An approach to the multi-path routing problem close to our approach is presented in Johansson et al. (2006) where the authors achieve the optimal network performance by a centralized simultaneous optimization of routing and power allocation, using convex optimization techniques. We differentiate from this work by considering decentralized solutions. The issue of joint optimization of routing and power allocation has also been tackled in Chiang (2005), giving birth to the problem of lifetime optimization. In fact, in order to maximize the lifetime of a WCN, one needs to uniformly distribute the power consumption rate of each node, as well as minimize the overall transmission power. In Toh, C.-K. (2001), the author shows that these two objectives cannot be satisfied simultaneously by a routing algorithm based (1) only on the minimization of the total power, (2) only 
on the minimization of battery consumption, or (3) only on the minimization of maximum battery consumption. A routing algorithm that consists of a tradeoff between a protocol that maximizes the lifetime of each node and a protocol that uses the battery fairly is proposed in Toh, C.-K. (2001).

The contribution of this paper is proposing a mathematical and simulative framework for applying a decentralized algorithm for dynamic multi-path routing control over a multi-hop network, in order to achieve the aforementioned power minimization objectives. Objective (1) requires a minimization of the sum over all nodes of the local transmission power; objective (2) requires a minimization of the sum over all nodes of the local battery discharge; objective (3) requires minimization of the maximum local transmission power among all nodes. The optimal routing policy for objectives (1) and (2) generally provides a single-path solution, which minimizes power transmission or battery consumption: for this reason, we will address without loss of generality only objective (1). The optimal routing policy for objective (3) generally provides a spread multi-path solution, which uniformly allocates power consumption over all the nodes. The optimization algorithm that we use in this paper is decentralized: we will show that it is robust with respect to time-varying transmission channel gain and node failures, and only requires transmission of local information between neighbor nodes. Moreover, the mathematical framework we propose in compatible with industrial specifications for multi-hop wireless networks, such as WirelessHART and ISA-100.

The paper is organized as follows. In Section 2 we illustrate the scheduling and routing WirelessHART specification in order to propose a mathematical framework of designing scheduling and routing policies, which is compliant with general time triggered specifications over multi-hop networks (e.g. WirelessHART, ISA-100). In Section 3 we define the decentralized optimization problems for objectives (1) and (3). In Section 4 we introduce our simulative environment (OMNeT ++ and MATLAB) and provide simulation results. Conclusions are offered in Section 5.

\section{MODELS FOR SCHEDULING AND ROUTING}

In this section we introduce the Network layer (routing) and the MAC layer (communication scheduling) of the WirelessHART specification. Let $\mathcal{V}$ be the set of wireless nodes of the network. Routing can be implemented in two configurations: graph routing and source routing. Graph routing provides, for a given destination node, a set of paths connecting all nodes of the network to the destination node as an acyclic directed graph associated to the destination node. In a properly configured network, and when permitted by the radio connectivity between nodes, all nodes must have at least two nodes in the graph through which they may send packets (ensuring redundancy and enhancing reliability). A typical routing graph for graph routing is illustrated in Figure 1. In source routing, only one path is associated to a given destination node. For this reason, source routing is much less reliable than graph routing, and the WirelessHART specification does not recommend to use it for control purposes.

Definition 1. Given a destination node $v_{d}$, we define the associated routing graph by an acyclic directed graph $\mathcal{G}_{v_{d}}=(\mathcal{V}, \mathcal{E})$, where the set of vertices $\mathcal{V}=1, \ldots,|\mathcal{V}|$ models the set of nodes, and the set of directed edges $\mathcal{E}=1, \ldots,|\mathcal{E}|$ models wireless communication directed links allowed by the routing graph.

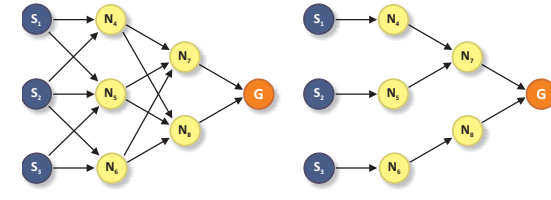

Fig. 1. Graph (left) and source (right) routing for the destination node $\mathrm{G}$ (gateway).

WirelessHART access to the channel is time slotted (HART Communication Foundation (2007)), where each slot has a time duration given by $\Delta=10 \mathrm{~ms}$. A finite sequence of $\Pi$ adjacent time slots forms a frame (called superframe in WirelessHART, Figure 2), which is repeated periodically with time duration $T=\Pi \Delta$. Slots of a frame can be either dedicated to one node or shared by several nodes: dedicated slots use TDMA for medium access, while shared slots use CSMA/CA for medium access. We will consider in our framework frames that have only dedicated slots with TDMA, and not shared slots. Latency requirements are addressed by scheduling the communication in such a way that packets will reach their destination in time, considering multiple hops, possible retransmissions, and alternate routes through the network.

Definition 2. Given a routing graph $\mathcal{G}=(\mathcal{V}, \mathcal{E})$, we define a scheduling policy as a string $\eta:\{1, \ldots, \Pi\} \rightarrow \mathcal{E}$, which associates to each time slot a communication link between a transmitting and a receiving node.

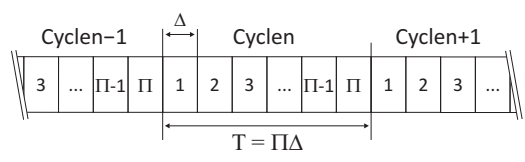

Fig. 2. Superframe structure.

A fundamental feature that characterizes WirelessHART is the interaction between the scheduling and the routing. According to the specification, for each frequency channel a schedule for the frame must be defined, where for each slot only one node is allowed to transmit and only a node is allowed to receive. The schedule must be designed so that all sensor data reaches the destination node (e.g. the controller). We recall that, according to the routing graph, each node has at least two neighbor choices to route a packet for any destination node. Moreover, in order to allow each node to choose a routing path according to a local decision algorithm, it is necessary to schedule the nodes' transmissions so that any path can be used, namely so that each node can locally decide the next destination for routing a packet among the choices given by the routing graph. This means that such a definition of the frame does not deterministically characterize the routing and thus the data flow in the network. The following example aims to clarify this concept, that is crucial for interpreting the semantics of data flow associated to transmission scheduling and graph routing.

Example: Consider the set of nodes $\mathcal{V}=\left\{S_{1}, N_{2}, N_{3}, G\right\}$, where the source node $S_{1}$ needs to route a packet to the destination node $G$. Let the routing graph associated to the destination node $G$ be $\mathcal{G}=\{\mathcal{V}, \mathcal{E}\}$, where $\mathcal{E}=\left\{\left(S_{1}, N_{2}\right),\left(S_{1}, N_{3}\right),\left(N_{2}, G\right),\left(N_{3}, G\right)\right\}$ as illustrated in Figure $3(\mathrm{a})$. Node $S_{1}$ has two choices for routing data, namely transmit data to node $N_{2}$ or to node $N_{3}$. To allow this local choice of node $S_{1}$, we need to schedule data transmission both for the pair $\left(S_{1}, N_{2}\right)$ and the pair $\left(S_{1}, N_{3}\right)$. Moreover, to allow data transmission to destination node $G$ both when node $N_{2}$ or 


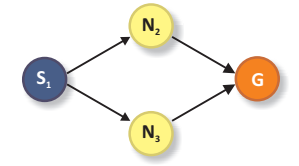

(a) Routing graph from node $S_{1}$ to $G$

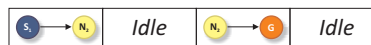

(c) Effective schedule if $S_{1}$ routes data via $N_{2}$.

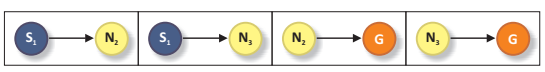

(b) Frame schedule. (d) Effective schedule if $S_{1}$ routes data via $N_{3}$.
Fig. 3. Interaction between scheduling and routing.

node $N_{3}$ has been involved in the routing, we need to schedule data transmission both for the pair $\left(N_{2}, G\right)$ and the pair $\left(S_{3}, G\right)$, as illustrated in Figure 3(b). Formally, the associated scheduling will be given by the string $\eta=\left\{\left(S_{1}, N_{2}\right),\left(S_{1}, N_{3}\right),\left(N_{2}, G\right),\left(N_{3}, G\right)\right\}$. This example clearly shows that a scheduling is not associated to a deterministic data flow in the network, but it is associated to a set of possible data flows that depend on the routing graph, and to local decisions of the nodes (e.g. because of failure of nodes and/or transmission errors). In Figures $3(\mathrm{c})$ and $3(\mathrm{~d})$ we illustrate the frame schedule and the two possible schedules that can effectively occur in the network in each frame, according to the decision of the source node $S_{1}$.

\section{DECENTRALIZED POWER AND ROUTING OPTIMIZATION}

Without loss of generality, we consider the case when $\mathcal{B}$ bytes have to be transmitted, for each frame, from a source node $v_{s}$ to a destination node $v_{d}$, e.g. from a sensor to a controller. We recall that $\Pi$ is the number of time slots of frame, and $T$ is the time duration of each time slot. Let $\mathcal{G}=(\mathcal{V}, \mathcal{E})$ be a given routing graph associated to the destination node $v_{d}$, and let $\eta:\{1, \ldots, \Pi\} \rightarrow E$ be a given periodic schedule. We recall that $\mathcal{V}$ is an acyclic directed graph, and that $\eta$ is defined allowing each node to route data to each path of the routing graph. For each node $v \in \mathcal{V}$, we denote by $\operatorname{inc}(v)=\left\{\left(v^{\prime}, v\right) \in \mathcal{E}: v^{\prime} \in \mathcal{V}\right\}$ the set of incoming edges to $v$, by $\operatorname{out}(v)=\left\{\left(v, v^{\prime}\right) \in \mathcal{E}: v^{\prime} \in \mathcal{V}\right\}$ the set of outgoing edges from $v$, and by $\mathcal{N}(v)=\left\{v^{\prime} \in \mathcal{V}\right.$ : $\left(v, v^{\prime}\right) \in \mathcal{E}$ or $\left.\left(v^{\prime}, v\right) \in \mathcal{E}\right\}$ the set of neighbor nodes of $v$.

We define an optimization variable $w \in\left(\mathbb{R}^{+}\right)^{|E|}$, where each component $w_{e}, e \in E$ models the data flow on link $\mathrm{e}$ during the associated time slot $\eta^{-1}(e)$. The routing graph $\mathcal{G}$ introduces constraints for the variable $w$ : more precisely, given any node $v \in \mathcal{V} \backslash\left\{v_{s}, v_{d}\right\}$, we require that for each frame the data sent to the outgoing links must be equal to the data received from the incoming links. For the source node $v_{s}$, which only has outgoing links, the data sent for each frame to the outgoing links must be equal to $\mathcal{B}$, since all sensing information is required to be eventually dispatched by $v_{s}$. For the destination node $v_{d}$, which only has incoming links, the data received for each frame from the incoming links must be equal to $\mathcal{B}$, since all sensing information is required to be eventually received by $v_{d}$. It is easy to verify that this last constraint is redundant, and is thus ignored in what follows. Formally, we define a constraint $w \in W$, where:

$$
\begin{aligned}
W=\left\{w \in\left(\mathbb{R}^{+}\right)^{|E|}\right. & : \sum_{e \in \operatorname{out}\left(v_{s}\right)} w_{e}=\mathcal{B}, \\
\sum_{e \in \operatorname{inc}(v)} w_{e} & \left.=\sum_{e \in \operatorname{out}(v)} w_{e}, \forall v \in \mathcal{V} \backslash\left\{v_{s}, v_{d}\right\}\right\} .
\end{aligned}
$$

For each link $e \in \mathcal{E}$ we define the transmission power needed to send a data flow $w_{e}$ on link $e$ in the time slot $\eta^{-1}(e)$ by applying the Shannon-Hartley Theorem Shannon (1949): $C_{e} \leq B_{e} \log _{2}\left(1+\mathrm{SNR}_{e}\right)$, where for each link $e, C_{e}$ is the post-correction effective channel capacity in bits per second, $B_{e}$ is the raw channel capacity in hertz (the bandwidth), $\mathrm{SNR}_{e}=\frac{P_{e}}{N_{e}}$ is the signal-to-noise ratio, $P_{e}$ is the transmission power, and $N_{e}$ is the noise power. Since the data flow $w_{e}$ must be transmitted within a single time slot, then it is necessary that $\frac{w_{e}}{T} \leq C_{e}$, with $T$ the time slot time duration. Thus, we assume that, given a data flow $w_{e}$ to be sent on link $e$, a node will use the minimum transmission power:

$$
p_{e}=N_{e}\left(2^{\frac{w_{e}}{B_{e} T}}-1\right) \text {. }
$$

Power Sum Optimization. In order to minimize the total power consumption in a network, we define a global optimization problem that minimizes the sum of the transmission power on all links:

$$
\begin{aligned}
& \operatorname{minimize} f(w)=\sum_{v \in \mathcal{V}} p_{v}=\sum_{e \in \mathcal{E}} N_{e}\left(2^{\frac{w_{e}}{B_{e} T}}-1\right), \\
& \text { subject to } w \in W,
\end{aligned}
$$

where $p_{v}=\sum_{e \in \text { out }(v)} p_{e}$. This optimization problem can be easily solved using convex optimization algorithms. Notice that the constraint $w \in W$ is given by the routing graph, which is available in each node. On the contrary, the cost function $f(w)$ contains information on the channel parameters $B_{e}, N_{e}$ for each link $e \in \mathcal{E}$. Since it is not realistic to assume that each node has knowledge of the channel parameters of any link, it is not possible for each node to locally solve the problem (1).

To overcome this issue, we address the above problem in a decentralized setting. For each node $v \in \mathcal{V}$, we define $\tilde{w}_{v}$, $\tilde{p}_{v}$, and $\tilde{w}_{e, v}$ respectively as the local estimates of $w, p_{v}$, and $w_{e}$. We solve the optimization problem that minimizes the sum of the transmission power of all outgoing links from node $v$ :

$$
\begin{aligned}
& \operatorname{minimize} f_{v}\left(\tilde{w}_{v}\right)=\tilde{p}_{v}=\sum_{e \in \operatorname{out}(v)} N_{e}\left(2^{\frac{\tilde{w}_{e, v}}{B_{e} T}}-1\right), \\
& \text { subject to } \tilde{w}_{v} \in W .
\end{aligned}
$$

In this case the cost function $f_{v}\left(\tilde{w}_{v}\right)$ contains information on the channel parameters $B_{e}$ and $N_{e}$ for each link $e \in \operatorname{out}(v)$, which are available locally in each node. Thus each node is able to locally solve the problem (2).

In order to solve (1) by locally solving (2), we use in each node the optimization subgradient algorithm proposed in Ram, S.S. et al. (2009). This algorithm can be locally applied to solve the problem (2), and only requires that each node $v$ has information of the vector $\tilde{w}_{v^{\prime}}$, for each $v^{\prime} \in \mathcal{N}(v)$. Since $f(w)=\sum_{v \in \mathcal{V}} f_{v}\left(\tilde{w}_{v}\right)$, it guarantees that any of the optimal solutions $\bar{w}_{v_{1}}, \ldots, \bar{w}_{v_{|\mathcal{V}|}}$ of $(2)$ converges to the optimal solution $\bar{w}$ of (1).

Maximum Power Optimization. In order to homogeneously share power consumption among all links, we define a global optimization problem that minimizes the maximum of the transmission power on all links:

$$
\begin{aligned}
& \operatorname{minimize} f(w)=\max _{e \in E}\left\{p_{e}\right\}, \\
& \text { subject to } w \in W .
\end{aligned}
$$

As discussed in the above section, we need to address the above problem in a decentralized setting. For each node $v \in \mathcal{V}$, we define a local optimization problem that minimizes the maximum among all neighbor nodes of the 
differences between the power consumption of $v$ and the power consumption of the neighbor node:

$$
\begin{aligned}
& \operatorname{minimize} f_{v}\left(\tilde{w}_{v}\right)=\max _{v^{\prime} \in \mathcal{N}(v)}\left\{\tilde{p}_{v}-\tilde{p}_{v^{\prime}}\right\}, \\
& \text { subject to } \tilde{w}_{v} \in W .
\end{aligned}
$$

In this case the cost function $f_{v}\left(\tilde{w}_{v}\right)$ contains information on the channel parameters $B_{e}$ and $N_{e}$ for each link $e \in \operatorname{out}(v)$ and for each link $e \in \operatorname{out}\left(v^{\prime}\right), \forall v^{\prime} \in \mathcal{N}(v)$, which are available locally to each node. Thus each node is able to locally solve the problem (4).

In order to solve (3) by locally solving (4), we use the same approach illustrated in the above Section. In this case, however, the global cost function $f(w)$ is not equal to the sum $\sum_{v \in \mathcal{V}} f_{v}\left(\tilde{w}_{v}\right)$ of the local cost functions. For this reason, any of the optimal solutions $\bar{w}_{v_{1}}, \ldots, \bar{w}_{v_{|\mathcal{V}|}}$ of (2) does not converge to the optimal solution $\bar{w}$ of (3), but they converge to a solution which spreads power consumption among nodes in a sub-optimal way. Defining an algorithm such that the optimal solutions $\bar{w}_{v_{1}}, \ldots, \bar{w}_{v_{|\mathcal{V}|}}$ converge to the optimal solution $\bar{w}$ of the optimization problem (3) is a theoretical problem that we will address in future work.

\section{SIMULATION RESULTS}

We used a hybrid simulation framework to implement our improved decentralized optimization algorithm, consisting of two different environments: one for performing convex optimization of the routing variables and the other for the network simulation. The convex optimization has been implemented in Matlab, and exploits the CVX package for convex optimization Grant and Boyd (March 2008). The optimization routines which lead the routing policies are called back from the Matlab environment by each node of the network simulator implemented in OMNeT++ (www.omnetpp.org). The Objective Modular Network Testbed in $\mathrm{C}++(\mathrm{OMNeT}++)$ is an objectoriented modular discrete event network simulator. OM$\mathrm{NeT}++$ is an appropriate simulation environment because it is a public-source, component based, modular and openarchitecture with strong GUI (Graphical User Interface) support and an embeddable simulation kernel. Moreover it is suitable to be used in modeling and simulation of multi-hop routing protocols in Wireless Sensor Networks as shown in Kacso, A.F. and Wismuller (2009). OM$\mathrm{NeT}++$ model consists of simple modules and compound modules. Simple modules are atomic elements in the module hierarchy: they can not be divided any further, and their most frequent task is sending and receiving messages. Messages can be sent either via output gates, or directly to another module. Gates are the input and output interfaces of modules, and they can be linked with connections. To each connection properties can be assigned, such as propagation delay, data rate and bit error rate. In our work, each wireless communication node is a simple module which decides its decentralized routing policy by collecting the data coming from its one-hop neighborhood, by calling the Matlab optimization routine which implements a decentralized, constrained subgradient algorithm. These nodes are connected by delay channels and their layout is defined by the NED language, which can be edited by both text and the GUI. We set up a test bed (depicted in Figure $4(\mathrm{a})$ ) consisting of a network of $N=6$ nodes, $m=5$ optimizing nodes, and $n=6$ links to test our algorithm. We defined two different scenarios: in the first one we show that the algorithm actually converges from any of the three different initial conditions toward the reference optimal configuration (which has been computed by means of a centralized convex optimization), while in the second scenario we show how fast the routing algorithm responds to the occurrence of a fault in a link, and converges to the new optimal configuration. We set up a second test bed (depicted in Figure 7(a)) consisting of a network of $N=11$ nodes, $m=10$ optimizing nodes and $n=26$ links to test our algorithm over a more complex network involving less intuitive optimization solutions, with a simple scenario in which the task consists in detecting a weak link located in the terminal stage of the network, and so potentially invisible to the nodes that are in the leading stages. Finally, for each of the possible scenarios depicted above, we solved the routing problem by means of the introduced optimization objectives.

First Test Bed: 6 Nodes Network. We consider the

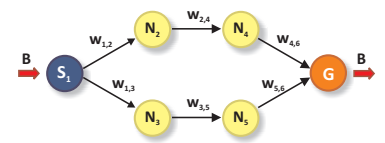

(a) Layout of the 6 nodes test

bed

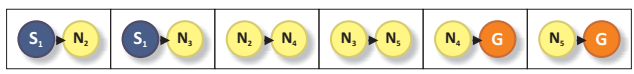

(b) Scheduling of the 6 nodes network

Fig. 4. Layout and scheduling of the first test bed.

network shown in Figure 4(a), scheduled as depicted in Figure 4(b), working in four different scenarios each one consisting of a batch of three simulations. For the first batch of simulations we used the algorithm optimizing the power sum with a single value of the step size $\alpha^{\text {sum }, 6}=0.1$ and three different initial routing configurations. The test bed has been conceived with the purpose of showing that the presence of a weaker link (from node $N_{4}$ to gateway $G$ ) in one of the two possible paths from the sensor $S_{1}$ to the gateway $G$ should hijack the traffic toward the other path, as the centralized solution suggests: $\bar{w}^{\text {sum }, 6}=$ $\left[\begin{array}{llllll}0 & 10 & 0 & 10 & 0 & 10\end{array}\right]^{\top}$, where the amount of traffic to transfer from sensor $S_{1}$ to gateway $G$ is $\mathcal{B}=10$. This should happen independently by the initial condition of the traffic distribution, so we picked up three significative values of the initial routing configuration, considering the results of the centralized optimization $\bar{w}^{\text {sum }, 6}$. The chosen initial conditions are: $w_{0, \text { best }}^{\text {sum } 6}=\left[\begin{array}{llllll}0 & 10 & 0 & 10 & 0 & 10\end{array}\right]^{\top}, w_{0, \text { medium }}^{\text {sum }, 6}=$ $\left[\begin{array}{llllll}5 & 5 & 5 & 5 & 5 & 5\end{array}\right]^{\top}, w_{0, \text { worst }}^{\text {sum, } 6}=\left[\begin{array}{llllll}10 & 0 & 10 & 0 & 10 & 0\end{array}\right]^{\top}$. As we can see, in $w_{0, \text { best }}^{\text {sum }, 6}$ the initialization is the best possible one since it is equal to the optimum $\bar{w}^{\text {sum, } 6}$. In $w_{0, \text { medium }}^{\text {sum, } 6}$ the initial condition distributes the traffic evenly along the two possible paths. The last initial condition $w_{0, w o r s t}^{\text {sum }}$ is the worst possible one, since it routes all the traffic through the weak link. The results of this first batch of three simulations are reported in Figure 5. In the second scenario, in correspondence of three different values of the steps size, we tested the robustness of our routing approach by considering a steady state configuration where the routing is the optimum $\left(w_{0, \text { best }}^{\text {sum }}, \mathbf{f}\right)$, and then introducing a fault in the terminal link of the preferred path (namely in the link from node $N_{5}$ to gateway $G$ ) in correspondence of the $20^{\text {th }}$ iteration, with the aim of verifying how fast our decentralized algorithm responds to external disturbances and converges to the new optimum. To better explain the influence of the step size onto the rate of convergence 


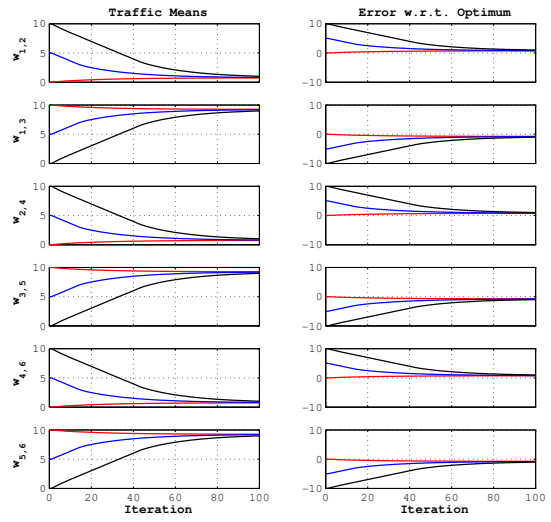

Fig. 5. Network with 6 nodes, minimizing sum of powers with initial routing policy $w_{0, \text { best }}^{\text {sum }, 6}$ (in red), $w_{0, \text { medium }}^{\text {sum } 6}$ (in blue), and $w_{0, w o r s t}^{\text {sum, } 6}$ (in black).

and the convergence error, we conduced three simulations with $\alpha_{1}^{\text {sum }, 6}=0.01, \alpha_{2}^{\text {sum, } 6}=0.1$ and $\alpha_{3}^{\text {sum }, 6}=0.5$. The new optimum routing policy after the fault occurs is given by: $\quad \bar{w}^{\text {sum }, 6, f a u l t}=\left[\begin{array}{llllll}10 & 0 & 10 & 0 & 10 & 0\end{array}\right]^{\top}$, so we expect that, starting at the $20^{\text {th }}$ iteration the algorithm will converge to a solution in which all the traffic is hijacked to the upper path. The results of this second batch of three simulations are reported in Figure 6 . In the third scenario we used the

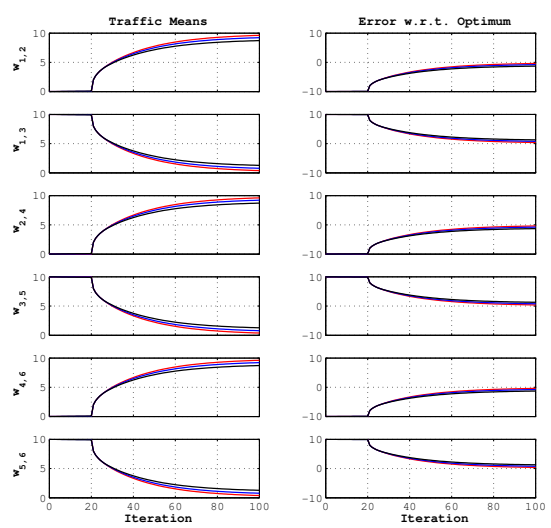

Fig. 6. Network with 6 nodes, minimizing sum of powers, with fault at iteration 20 for different values of the step size: $\alpha_{1}^{\text {sum }, 6}=0.01$ (in red), $\alpha_{2}^{\text {sum }, 6}=0.05$ (in blue), and $\alpha_{3}^{\text {sum, } 6}=0.5$ (in black).

algorithm optimizing the power max with a single value of the step size $\alpha^{\max , 6}=0.1$ and three different initial routing configurations. The test bed has been conceived with the purpose of showing that the presence of a weaker link in one of the two possible paths (from node $N_{4}$ to gateway $G$ ) from the sensor $S_{1}$ to the gateway $G$ should not completely hijack the traffic towards the other path, as the centralized solution suggests: $\bar{w}^{\max , 6}=\left[\begin{array}{lllll}1 & 9 & 1 & 9 & 1\end{array}\right]^{\top}$, supposing that the amount of traffic to switch from sensor $S_{1}$ to gateway $G$ is 10 . This should happen independently by the initial condition of the traffic distribution, so we picked up three significative values of the initial routing configuration also considering the results of the centralized optimization $w_{o p t}^{\max , 6}$. The chosen ini-

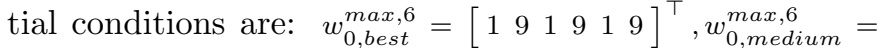
$\left[\begin{array}{llllll}5 & 5 & 5 & 5 & 5 & 5\end{array}\right]^{\top}, w_{0, \text { worst }}^{\max , 6}=\left[\begin{array}{llllll}10 & 0 & 10 & 0 & 10 & 0\end{array}\right]^{\top}$. As we can see, in $w_{0, \text { best }}^{\max , 6}$ the initialization is the best possible one since it is equal to $\bar{w}^{\max , 6}$. In $w_{0, \text { medium }}^{\max , 6}$ the initial condition distributes the traffic evenly along the two possible paths. The last initial condition $w_{0, \text { worst }}^{\max , 6}$ routes all the traffic through the weak link. The convergence results of this third batch of simulations are similar to those of the first batch, with convergence value $\bar{w}^{\max , 6}$. In the fourth scenario, in correspondence of three different values of the steps size, we tested the robustness of our routing approach letting it work without any problem with the best initial condition of the previous scenario $\left(w_{0, \text { best }}^{\max , 6}\right)$ for the first 20 iterations and introducing then a fault in correspondence of the $20^{t h}$ iteration in the terminal link of the preferred path (namely in the link from node $N_{5}$ to gateway $G$ ) in order to show how fast our routing algorithm converges to the new optimum. To better explain the influence of the step size onto the rate of convergence and the convergence error whereby the subgradient method converges, we conduced three simulations with three different values of the parameter: $\alpha_{1}^{\max , 6}=0.01, \alpha_{2}^{\max , 6}=0.1$ and $\alpha_{3}^{\max , 6}=0.5$. The new optimum routing policy calculated after that the fault occurs is: $\bar{w}^{\max , 6, \text { fault }}=\left[\begin{array}{llllll}10 & 0 & 10 & 0 & 10 & 0\end{array}\right]^{\top}$, so we expect that after 20 iterations in which all the traffic is routed along the lower path, the algorithm will converge to a solution in which all the traffic is hijacked to the upper path. The convergence results of this fourth batch of three simulations are similar to those of the second batch, with convergence value $\bar{w}^{\max , 6, \text { fault }}$.

Finally, we realized an OMNeT ++ implementation of this particular network in which the functions for the optimization of the routing were recalled directly from inside the $\mathrm{OMNeT}++$ environment by leveraging the Matlab Engine.

Second Test Bed: 11 Nodes Network. We consider

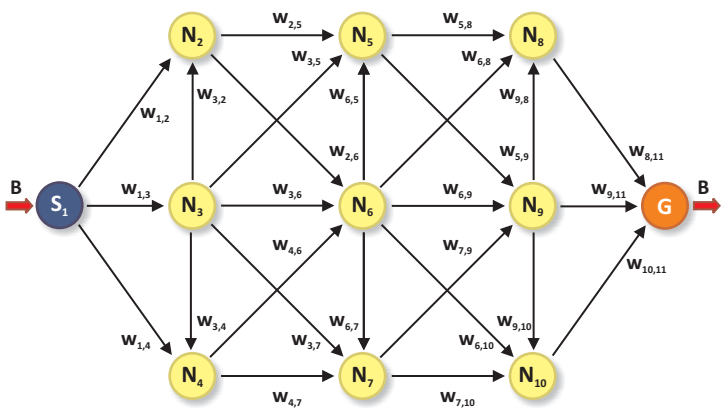

(a) Layout of the 11 nodes test bed

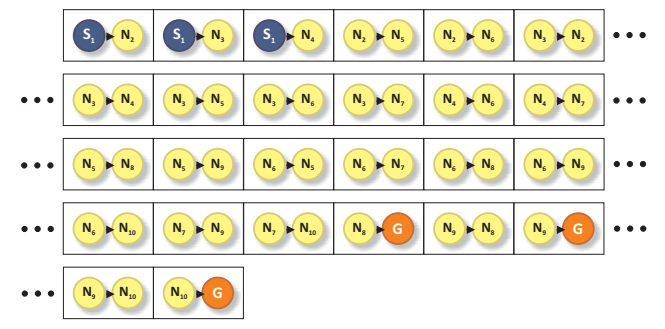

(b) Scheduling of the 11 nodes network

Fig. 7. Layout and scheduling of the second test bed.

the network shown in Figure 7(a), scheduled as depicted 
in Figure 7(b). We used the algorithm minimizing the sum of powers of the nodes with a single value of the step size $\alpha^{\text {sum,11 }}=0.25$ starting from a non-optimum initial routing configuration. In this network we check the behaviors of the last three links which are directly connected to the gateway node $G$. The test bed has been created with the purpose of showing that the algorithm will penalize the weaker link (namely the link from node $N_{8}$ to the gateway $G$ ) routing all the traffic through the other two links of the terminal stage of the network, as the centralized optimum solution suggests: $\bar{w}^{\text {sum }, 11}[8, G]=$ $0, \bar{w}^{\text {sum }, 11}[9, G]=15, \bar{w}^{\text {sum }}, 11[10, G]=15$, where the amount of traffic to transfer from sensor $S_{1}$ to gateway $G$ is $\mathcal{B}=30$. The simulation algorithm is initialized with a routing policy which distributes evenly the traffic among the links of the terminal stage of the network: $w_{0}^{\text {sum, } 11}[8, G]=$ $10, w_{0}^{\text {sum }, 11}[9, G]=10, w_{0}^{\text {sum }, 11}[10, G]=10$. The results of this first simulation are reported in Figure 8. As we can see, the number of iterations needed to converge toward the optimal solution is around one thousand, while in the first network is around one hundred. This is due to the fact that the number of optimizing nodes in this second test bed is greater than the number of optimizing node of the previous test bed. In the second scenario we used

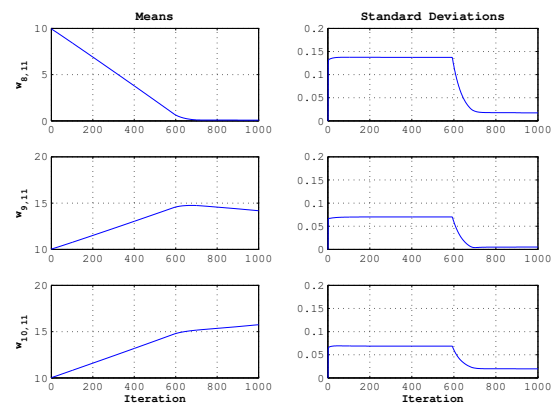

Fig. 8. Network with 11 nodes, minimizing the sum of powers with initial routing policy $w_{0}^{\text {sum,11. }}$.

the algorithm minimizing the maximum power among the nodes with a single value of the step size $\alpha^{\max , 11}=$ 0.25 and starting from a non-optimum initial routing configuration. The test bed has been conceived with the purpose of showing that the presence of a weaker link among the three links of the terminal stage of the network should not completely hijack the traffic towards the central link of the final stage, as the centralized solution suggests: $\bar{w}^{\max , 11}[8, G]=1.5, \bar{w}^{\max , 11}[9, G]=13.5, \bar{w}^{\max , 11}[10, G]=15$, supposing that the amount of traffic to switch from sensor $S_{1}$ to gateway $G$ is $\mathcal{B}=30$. We picked up the same initial condition of the previous simulation: $w_{0}^{\max , 11}[8, G]=$ $10, w_{0}^{\max , 11}[9, G]=10, w_{0}^{\max , 11}[10, G]=10$. The convergence results of this second simulation are similar to those of the previous one. However, as we expected, even though the traffic is deviated towards the two healthy links of the last stage of the network, there is a little amount of it that is still delivered through the weak link of the terminal stage. Moreover, as noticed in the previous simulation, the number of iterations necessary to the algorithm to converge to the optimal centralized solution is around one thousand.

\section{CONCLUSIONS AND FUTURE WORK}

We proposed a mathematical and simulative framework for dynamic routing control in order to minimize power consumption objectives, which is compliant with the WirelessHART specification for multi-hop wireless control networks. To achieve this goal we proposed the use of a decentralized optimization algorithm, and we showed by simulation its robustness with respect to time-varying transmission channels and to node failures.

\section{ACKNOWLEDGEMENTS}

The authors gratefully thank Maria Gabriella Di Benedetto, Luca De Nardis and Carlo Fischione for interesting discussions on routing algorithms.

\section{REFERENCES}

Bertsekas, D. and Gallager, R. (1987). Data Networks. Prentice Hall.

Chiang, M. (2005). Balancing Transport and Physical Layers in Wireless Multi-hop Networks: Jointly Optimal Congestion Control and Power Control. IEEE Journal on Selected Areas in Communications.

Grant, M. and Boyd, S. (March 2008). CVX: Matlab Software for Disciplined Convex Programming. http://stanford.edu/boyd/cvx.

HART Communication Foundation (2007). TDMA DataLink Layer Specification. HCF SPEC 075 Revision 1.0.

Hazarika, S., Kurose, J., and Goeckel, D. (2004). An Approach to Minimal Power Routing and Scheduling in Wireless Ad-Hoc Networks. Technical report, UMass CMPSCI Technical Report.

Hedrick, C. (1988). Routing Information Protocol. Internet Request for Comments 1058.

Johansson, M., Xiao, L., and Boyd, S. (2006). Simultaneous Routing and Power Allocation in CDMA Wireless Data Networks. In Proceedings of the IEEE International Conference on Communications.

Johnson, D.B. and Maltz, D.A. (1996). Dynamic Source Routing in Ad-Hoc Wireless Networks. Mobile Computing.

Kacso, A.F. and Wismuller, R. (2009). Modeling and Simulation of Multi-hop Routing Protocols in Wireless Sensor Networks. In Proceedings of the $5^{\text {th }}$ International Conference on Wireless and Mobile Communications.

Moy, J. (1991). OSPF. Internet Request for Comments 1247.

Mueller, S., Tsang, R.P., and Ghosal, D. (2003). Multipath Routing in Mobile Ad-Hoc Networks: Issues and Challenges. In MASCOTS Tutorials.

Perkins, C.E. and Royer, E.M. (1999). Ad-hoc OnDemand Distance Vector Routing. Proceedings of the $2^{\text {nd }}$ IEEE Workshop on Mobile Computing Systems and Applications.

Ram, S.S., Nedic, A., and Veeravalli, V.V. (2009). Distributed subgradient projection algorithm for convex optimization. In Proceedings of the IEEE International Conference on Acoustics, Speech and Signal Processing, 3653-3656.

Shannon, C. (1949). The Mathematical Theory of Information. University of Illinois Press.

Song, J., Han, S., Mok, A.K., Chen, D., Lucas, M., Nixon, M., and Pratt, W. (2007). WirelessHART: Applying Wireless Technology in Real-Time Industrial Process Control. In Proceedings of the $13^{\text {th }}$ IEEE Real-Time and Embedded Technology and Applications Symposium.

Toh, C.-K. (2001). Maximum Battery Life Routing to Support Ubiquitous Mobile Computing in Wireless Ad Hoc Networks. IEEE Communications Magazine.

Zaumen, W.T. and Garcia-Luna-Aceves, J.J. (1998). Loop-Free Multi-path Routing Using Generalized Diffusing Computations. In INFOCOM (3). 